\title{
Middle latency auditory evoked potential index for prediction of post-resuscitation survival in elderly populations with out-of-hospital cardiac arrest
}

\author{
JUNYA TSURUKIRI, KATSUHIRO NAGATA, KENICHIRO KUMASAKA, KEIKO UENO, MASAHITO UENO
}

Department of Emergency and Critical Care Medicine, Tokyo Medical University Hachioji Medical Center

Corresponding author:

Junya Tsurukiri

Department of Emergency and Critical Care Medicine

Tokyo Medical University

1163 Tatemachi Hachioji-shi Tokyo, 193-0998, Japan

Phone: 81-42-665-5611, FAX: 81-42-665-5639

E-mail:junya99@tokyo-med.ac.jp

\section{ABSTRACT}

Background. Out-of-hospital cardiac arrest (OHCA) is associated with a high mortality rate in the elderly. Although most reports have investigated among elderly patients with OHCA until 1990s, non-invasive monitorings cannot presently predicted cerebral resuscitation during cardiopulmonary resuscitation (CPR). Findings of a previous study suggest that monitoring of middle latency auditory evoked potentials (MLAEP) during CPR could provide an indicator of effective post-resuscitation survival.

Objectives. We speculated that the MLAEP index (MLAEPi), measured in an emergency room, can predict post-resuscitation survival among elderly patients with OHCA.

Methods. This prospective study included 31 elderly patients aged $\geq 65$ years with OHCA who received basic life support (BLS) and did not achieve restoration of spontaneous circulation (ROSC) until arrival at the emergency center between December 2010 and December 2011. All patients were administered advanced cardiac life support (ACLS) in the emergency room. Initial MLAEPi was measured using an MLAEP monitor (aepEX plus ${ }^{\oplus}$, Audiomex, UK) during the first cycle of ACLS. Prediction of the post-resuscitation survival was investigated.

Results. Eight patients who achieved ROSC were admitted to our hospital and 23 did not achieve ROSC in the emergency room. Initial MLAEPi was significantly higher in patients with than without ROSC (median, 33 vs. $26, p=0.02$ ). Three survivors, among patients with ROSC, were discharged from our hospital (survivors) and 5 died during hospitalization (non-survivors). Initial
MLAEPi was significantly higher in survivors than in non-survivors (median, 35 vs. $28, \mathrm{p}=0.03$ ) or patients without ROSC (median, 35 vs. 26, $\mathrm{p}<0.01$ ).

Conclusions. MLAEPi satisfactorily denotes cerebral function and predicts postresuscitation survival in elderly populations.

Key words: cardiopulmonary resuscitation, basic life support, advanced cardiac life support, age, monitoring, critical care

\section{INTRODUCTION}

Out-of-hospital cardiac arrest (OHCA), which is associated with a high mortality rate, is one of the most common causes of death. One study found that the overall one month survival rate was $6.9 \%$ and the rate of good neurological outcome was $2.8 \%$ in elderly with OHCA. (1) Although most published reports in the 1990s and 2000s have investigated OHCA among elderly patients, non-invasive monitoring of elderly patients with OHCA cannot presently predict cerebral resuscitation during cardiopulmonary resuscitation (CPR). (2-4) They describe studies of adult populations during the early phase of post-resuscitation, but the findings of an animal study suggest monitoring of auditory evoked potentials (AEPs) during CPR could provide an indicator of effective resuscitation and post-resuscitation survival. (5-8) Moreover, a recent report suggests that the components of middle latency auditory evoked potentials (MLAEPs), which are derived from AEPs, might predict postresuscitation outcomes. (9)

Middle latency auditory evoked potentials can provide a good indicator of conscious- ness levels during anesthesia. (10) Data of AEPs or MLAEP are usually intermittently generated using large dedicated instruments. Analyzing waveforms of MLAEP might be challenging in an emergency room delivering emergency care. The MLAEPs monitor (aepEX plus ${ }^{\circ}$, Audiomex, Glasgow, Scotland, UK) is the first mobile MLAEPs monitor that is available for measurement of the depth of anesthesia. (11) It continuously generates an MLAEP index (MLAEPi), which is a dimensionless number scaled between 100 (wide awake) and 0 (no brain activity), with differences between successive segments of the curve constructed from its amplitude (figure 1).

We thus speculated that the initial MLAE$\mathrm{Pi}$, determined upon arrival at the emergency department (ED), can indicate cerebral function and predict post-resuscitation survival among elderly patients with OHCA.

\section{MATERIALS AND METHODS}

\section{Hospital and setting}

This study took place at the Emergency Center between December 2010 and December 2011. The ethics committee of the institution approved the study design, and informed consent was obtained from the patient's next of kin or a posteriori from the patients themselves, when possible, after emergency resuscitation, because monitoring was non-invasive and nonrandomized.

\section{Patients}

All calls concerning OHCA to call centers 


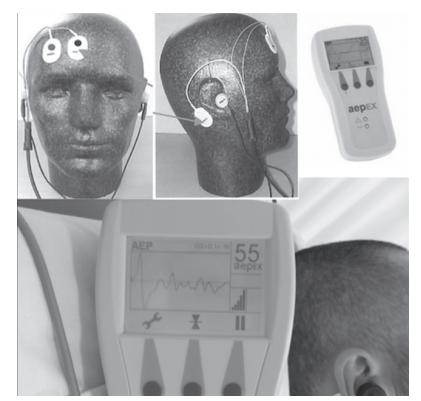

Figure 1. The aepEX equipment

were immediately transferred to the fire department rescue teams located closest to the victims. Paramedics immediately started basic life support (BLS) including external cardiac massage, airway management or ventilation and semi-automatic defibrillation. Japan is divided into distinct medical regions, and victims are dispatched to emergency centers at regional hospitals. Upon arrival at an emergency center, an emergency medical team, comprising paramedics, nurses, residents and senior physicians specialized in emergency medicine, provides advanced cardiac life support (ACLS) according to current resuscitation guidelines. (12) All elderly patients with OHCA received basic life support (BLS) from bystanders or paramedics within 1 $\mathrm{h}$ of collapse. We excluded patients aged younger than 65 years, those who arrived over one hour after collapse, those who achieved restoration of spontaneous circulation (ROSC) before arrival at hospital, bedridden patients and those with trauma, tympanic injury or terminal diseases.

We defined cardiac arrest as the absence of both spontaneous breathing and a palpable carotid pulse including asystole, pulseless electrical activity (PEA) or ventricular fibrillation (Vf)/ventricular tachycardia (VT). The present study included all patients with OHCA who received BLS from bystanders or paramedics within one hour of collapse and who did not achieve sequential ROSC until arrival at the emergency center. All patients did not receive any sedation or neuromuscular blockers before measurement of initial MLAEPi at the emergency rooms.

\section{Middle latency auditory evoked poten- tial index (MLAEPi)}

The MLAEPi was continuously calculated using the aepEX plus ${ }^{\circledast}$ from information provided by sensor electroencephalogram (EEG) electrodes affixed to the patient's middle (ground electrode) and right fore-

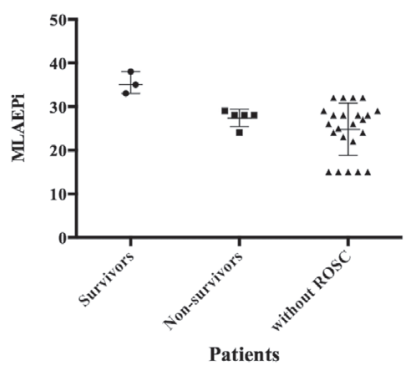

Figure 2. Initial middle latency auditory evoked potential index (MLAEPi) among survivors, non-survivors and patients without restoration of spontaneous circulation (ROSC). Boxes plotted groupwise indicate medians and interquartiles with extreme values as whiskers.

survivors vs. non-survivors $(p=0.03)$ and survivors vs. patients without ROSC $(p<$ 0.01 ).

head (active electrode) as well as the right mastoid (active electrode), after cleaning the skin with $70 \%$ isopropanol. An emergency medical physician also applied earphones to determine auditory stimuli. The AEP was elicited with bilateral click stimuli via earphones at an intensity of $<60$ $\mathrm{dB}$ for a nominal frequency of $6.9 \mathrm{~Hz}$. The detected AEPs are consecutively extracted from the raw EEG signal, which reflects the brainstem auditory-evoked potential and the MLAEP by the internal processor. The aepEX values are closely related to the AEP waveforms and are calculated as the sum of square roots of the absolute difference between every two successive 0.56 ms segments of the AEP waveforms. The aepEX continuously generates an MLAEP index (MLAEPi), which is a dimensionless number scaled between 100 (wide awake) and 0 (no brain activity).

\section{Intervention and data collection}

All patients with OHCA who did not show ROSC until arrival at the emergency center were then administered ACLS, and MLAEPi was immediately measured during the first cycle of ACLS. The measurement of MLAEPi was performed by 1 emergency physician (JT) at the ED and the data was blinded to other emergency physicians during resuscitation. The measurement of MLAEPi did not affect standard ACLS and post-resuscitation management procedures in the emergency rooms. In patients who showed ROSC, the MLAEPi was measured at ROSC and at $1 \mathrm{~h}$ after ROSC. The following patient characteristics were

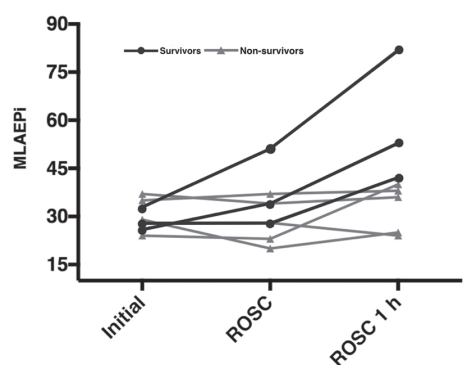

Figure 3. Changes of middle latency auditory evoked potential index (MLAEPi) among survivors and non-survivors.

retrieved from charts and electrocardiograms: age, gender, medical history, body temperature, blood values $(\mathrm{pH}$, lactate levels, and base excess), first recorded electrical rhythm by the EMS or bystanders, whether the OHCA was witnessed by a bystander or not, whether the bystander performed CPR or not, resuscitation intervals, whether or not the Emergency Medical Service (EMS) or bystander attempted defibrillation, as well as post-resuscitation outcomes at 3 months after OHCA. (13)

Statistical analysis

Data from all eligible patients were analyzed. Continuous variables are shown as median values with interquartile ranges. Between-group differences were statistically assessed using the Mann-Whitney Utest for continuous variables and Fisher's exact test for categorical variables using Prism version 5.0d (GraphPad Software, San Diego, CA, USA) as appropriate. Categorical variables were calculated as ratios (\%) of the frequency of occurrence. Statistical significance was indicated at the level of 0.05 .

\section{RESULTS}

\section{Clinical characteristics}

We identified 31 elderly patients with OHCA who received BLS in the pre-hospital setting. Among patients, 8 with ROSC were admitted to our intensive care unit (ICU) and 23 did not achieve ROSC upon resuscitation in the emergency room. Table 1 compares their baseline characteristics. There was no significant difference in the number of patients undergoing therapeutic hypothermia after resuscitation between the group with ROSC and nonROSC. Initial MLAEPi was significantly higher in patients with than without ROSC (median, 33 vs. 26, $\mathrm{p}=0.02$ ). 
Table 1. Clinical characteristics and comparison of patients with or without restoration of spontaneous circulation

\begin{tabular}{|c|c|c|c|c|}
\hline \multirow{2}{*}{ Clinical variables } & \multirow[b]{2}{*}{ Total $(\mathrm{n}=31)$} & \multicolumn{3}{|c|}{ Univariate analysis } \\
\hline & & $\operatorname{ROSC}(n=8)$ & non-ROSC $(n=23)$ & $p$ value \\
\hline Age (years)-median (IQR) & $76(70-81)$ & $79(76-82)$ & $76(70-80)$ & 0.16 \\
\hline Male, $n(\%)$ & $20(65)$ & $5(63)$ & $15(65)$ & 1.00 \\
\hline Tympanic temperature $\left({ }^{\circ} \mathrm{C}\right)$-median $(\mathrm{IQR})$ & $35.0(34.1-35.3)$ & $35.3(34.9-35.8)$ & $34.8(34.0-35.1)$ & 0.07 \\
\hline $\mathrm{pH}$-median (IQR) & $7.00(6.86-7.13)$ & $7.05(6.84-7.15)$ & $6.96(6.87-7.12)$ & 0.76 \\
\hline Lactate concentration (mmol/L) -median (IQR) & $11.7(8.8-16.5)$ & $10.0(8.3-15.2)$ & $12.9(10.1-16.5)$ & 0.51 \\
\hline Base excess -median (IQR) & $-11.5(-16.7--8.4)$ & $-10.1(-17.5--6.4)$ & $-12.7(-15.7--9.1)$ & 0.67 \\
\hline \multicolumn{5}{|l|}{ First recorded rhythm on arrival, $\mathrm{n}(\%)$} \\
\hline Asystole & $22(71)$ & $5(63)$ & $17(74)$ & 0.66 \\
\hline Pulseless electrical activity & $8(26)$ & $3(38)$ & $5(22)$ & 0.39 \\
\hline $\mathrm{VF}$ & $1(3)$ & 0 & $1(4)$ & 1.00 \\
\hline Witnessed cadiac arrest, $\mathrm{n}(\%)$ & $19(61)$ & $7(88)$ & $12(52)$ & 0.11 \\
\hline Bystander CPR, n (\%) & $18(58)$ & $5(63)$ & $13(57)$ & 1.00 \\
\hline Determined VF during out-of-hospital management, $\mathrm{n}(\%)$ & $5(16)$ & $2(25)$ & $3(13)$ & 0.58 \\
\hline \multicolumn{5}{|l|}{ Resuscitation intervals (min) -median (IQR) } \\
\hline To starting BLS & $7(3-8)$ & $6(4-6)$ & $7(2-9)$ & 0.44 \\
\hline To starting ACLS (Total, $\mathrm{n}=30 ; \mathrm{ROSC}, \mathrm{n}=7 ;$ non- $\mathrm{ROSC}=23$ ) & $36(29-40)$ & $31(24-36)$ & $36(30-41)$ & 0.43 \\
\hline Therapeutic hypothermia, $\mathrm{n}(\%)$ & $8(26)$ & $3(38)$ & $5(22)$ & 0.39 \\
\hline Initial MLAEPi -median (IQR) & $28(24-30)$ & $33(29-40)$ & $26(23-29)$ & 0.02 \\
\hline
\end{tabular}

auditory evoked potential index; ROSC, restoration of spontaneous circulation; VF, ventricular fibrillation."

With regard to survival, 3 patients who achieved ROSC were discharged from our hospital (survivors) but the other 5 died (non-survivors) during hospitalization. There was no significant difference in the number of patients undergoing therapeutic hypothermia after resuscitation between survivors and non-survivors (number, 2 vs. $6, \mathrm{p}=0.16$ ). Figure 2 shows the MLAEPi records of survivors, non-survivors and patients without ROSC. Initial MLAEPi was significantly higher in survivors than in non-survivors (median, 35 vs. $28, \mathrm{p}=$ 0.03 ) and patients without ROSC (median, 35 vs. $26, \mathrm{p}<0.01)$. Although initial MLAEPi and MLAEPi at achieving ROSC were not significantly different between survivors and non-survivors in the ED, the MLAEPi at $1 \mathrm{~h}$ after achieving ROSC was significantly different (median, 53 vs. 36, P $=0.04)$ (figure 3 ).

\section{DISCUSSION}

This study is the first to evaluate the MLAE$\mathrm{Pi}$ of elderly populations with OHCA in an emergency center. Our results showed that initial MLAEPi of survivors is significantly higher than in non-survivors or patients without ROSC. These results indicate that MLAEPi can detect cerebral function during $\mathrm{CPR}$ in the $\mathrm{ED}$ and might increase the certainties of a bad prognosis leading to earlier cessation of resuscitation in emergency centers among elderly populations with OHCA.

Out-of-hospital cardiac arrest, which is associated with a high mortality rate, is one of the most common causes of death. In a Japanese nationwide study among the elderly population, the overall one month survival rate was $6.9 \%$ and the rate of good neurological outcomes was $2.8 \%$, after OHCA. (1) Moreover, elderly populations with OHCA are growing, as well as increasing their life expectancies. Although a major goal for OHCA resuscitation is to improve the rate of post-resuscitation outcomes, effective indicators have not yet been established in the emergency setting. The value of AEP monitoring in patients with cardiac arrest is controversial and most published reports have been conducted during the early phase of post-resuscitation. (5-7) The findings of an animal study suggest that monitoring AEPs during CPR could provide an indicator of restored cerebral blood flow and thus effective resuscitation and post-resuscitative survival. (8) Moreover, the P50 components of the MLAEP are considered to be hippocampal CA3 pyramidal cells and the presence of P50 might predict post-resuscitation outcomes after resuscitation. (9) Our results confirmed these findings in the emergency setting even though few of our patients had post-resuscitation outcomes.

The MLAEP is derived from AEP, and it reflects the morphology of MLAEP curves. The aepEX plus identifies all brain stem and cortical components, especially positive $\mathrm{Pa}$ and negative $\mathrm{Nb}$ waves, of MLAEP after auditory stimuli, and the MLAEPi is calculated from consistent decreases in amplitude and increases in latency, resulting in individual waves within $144 \mathrm{msec}$. It is increasingly used as a measurement of both the level of anesthesia and cerebral function, instead of bispectral index (BIS) values in intensive care units (ICUs). (1417) To analyze waves in real time is diffi- cult during an emergency clinical situation using the MLAEP, which is usually obtained intermittently. However, the mobile battery operated aepEX monitor provides consistent assessment of the MLAEP during life-saving procedures while transporting patients within the hospital or to admitted patients. The MLAEPi was a better indicator of sedation depth than the BIS or any other EEG-based monitoring method, and $100 \%$ specificity was found for MLAE$P i$ values of 37 (for unconsciousness) and 61 (for being awake during anesthesia). $(15,16)$

Previous reports have confirmed that a witnessed arrest and Vf/VT rhythms are key prognostic factors for survival in elderly patients with cardiac arrest. $(2,18)$ However, most of our patients were already in asystole upon arrival at the emergency centers in our study. The appearance of Vf/VT rhythms was determined out-of-hospital in 5 patients, all of whom received automated external defibrillation. Thus, only patients with recurrent Vf/VT arrived at the emergency center. Although all survivors had a witnessed arrest in our study, there was no significant difference in the number of witnessed arrests between survivors and non-survivors (number, 3 vs. $16, \mathrm{p}=0.26$ ). Thus, a witnessed arrest did not have an influence on our outcomes in our small population. Furthermore, although postresuscitation care, including therapeutic hypothermia, is well known to affect the outcomes of comatose patients, we did not determine significant differences in the number of patients with a Glasgow Coma Scale (GCS) score $\leq 8$ undergoing therapeutic hypothermia. (19)

\section{LIMITATIONS}

This study has several limitations, the most notable being the number of patients. First, we did not monitor MLAEPi during BLS or ACLS in the pre-hospital setting. A recent report suggested that the presence of pre-hospital ROSC is one of the most important prognostic indicators of a favorable outcome for OHCA and survival rates after hospitalization are higher with, than without pre-hospital ROSC. (20) Thus, resuscitation efforts should focus on achieving pre-hospital ROSC. Secondly, we only obtained MLAEPi data during primary resuscitation and had no records from the early phase (within $24 \mathrm{~h}$ ) of post-resuscitation care. The purpose of this study was to assess MLAEPi monitoring for practical prognostication in an emergency setting. Thirdly, we did not routinely measure end- 
tidal carbon-dioxide (EtCO2) at our ED, thus we could not correlate the MLAEPi and $\mathrm{EtCO} 2$.

\section{CONCLUSIONS}

The present results indicate that initial MLAEPi, represented by simple numerical values upon presentation at emergency centers, helps to predict post-resuscitation outcomes in elderly populations with
OHCA. Larger studies are essential to further evaluate the role of MLAEPi monitoring of neurological outcomes in elderly populations with OHCA and advancement of approaches for pre-hospital resuscitation necessitates up-to-date information.

\section{REFERENCES}

1. Akahane M, Tanabe S, Koike S, Ogawa T, Horiguchi H, Yasunaga H, et al. Elderly out-of-hospital cardiac arrest has worse outcomes with a family bystander than a non-family bystander. Int J Emerg Med 2012 Nov;5(1):41. doi: 10.1186/1865-1380-5-41.

2. Mosier J, Itty A, Sanders A, Mohler J, Wendel C, Poulsen J, et al. Cardiocerebral resuscitation is associated with improved survival and neurologic outcome from out-of-hospital cardiac arrest in elders. Acad Emerg Med 2010 Mar;17(3):269-75. doi: 10.1111/j.15532712.2010.00689.x.

3. Bonnin MJ, Pepe PE, Clark PS Jr. Survival in the elderly after out-of-hospital cardiac arrest. Crit Care Med 1993 Nov;21(11):1645-51.

4. Van Hoeyweghen RJ, Bossaert LL, Mullie A, Martens P, Delooz HH, Buylaert WA, et al. Survival after out-of-hospital cardiac arrest in elderly patients. Belgian Cerebral Resuscitation Study Group. Ann Emerg Med 1992 Oct;21(10):1179-84.

5. Tiainen M, Kovala TT, Takkunen OS, Roine RO. Somatosensory and brainstem auditory evoked potentials in cardiac arrest patients treated with hypothermia. Crit Care Med 2005 Aug;33(8):1736-40.

6. Sakurai A, Kinoshita K, Moriya T, Utagawa A, Ebihara T, Furukawa M, et al. Reduced effectiveness of hypothermia in patients lacking the wave $\mathrm{V}$ in auditory brainstem responses immediately following resuscitation from cardiac arrest. Resuscitation 2006 Jul;70(1):528.

7. Westerén-Punnonen SM, Yppärilä H, Musialowicz T, Korhonen I, Hynynen M, Partanen J. Recovery of N100 component of auditory event-related potentials and EEG after cardiac arrest during propofol sedation. Br J Anaesth 2005 May;94(5):626-9.

8. Reid KH, Mullins ER, Iyer VG. Changes in brainstem auditory evoked response latency predict survival after CPR in a rat model of cardiac arrest and resuscitation. Resuscitation 1998 Jan;36(1):65-70.

9. Takai N, oda S, Sadahiro T, Nakamura M, Watanabe E, Takeishi, et al. Auditory evoked potential P50 as a predictor of neurological outcome in resuscitated cardiac arrest patients. J Clin Neurophysiol 2011 Jun;28(3):302-7.

10. Jildenstål PK, Hallén JL, Rawal N, Gupta A, Berggren L. Effect of auditory evoked potential-guided anaesthesia on consumption of anaesthetics and early postoperative cognitive dysfunction: a randomized controlled trial. Eur J Anaesthesiol 2011 Mar;28(3):213-9. doi: 10.1097/EJA.0b013e3283400dbb9.

11. Mantzaridis H, Kenny GN. Auditory evoked potential index: a quantitative measure of changes in auditory evoked potentials during general anaesthesia. Anaesthesia 1997 Nov;52(11):1030-6.

12. Nolan JP, Deakin CD, Soar J, Böttiger BW, Smith G; European Resuscitation Council. European resuscitation council guidelines for resuscitation 2005. Section 4. Adult advanced life support. Resuscitation 2005 Dec;67 suppl 1:S39-86.

13. Ajam K, Gold LS, Beck SS, Damon S, Phelps R, Rea TD. Reliability of the cerebral performance category to classify neurological status among survivors of ventricular fibrillation arrest: a cohort study. Scand J Trauma Resusc Emerg Med 2011 Jun;19:38. doi: 10/1186/1757-7241-19-38.

14. Slabu L, Escera C, Grimm S, Costa-Faidella J. Early change detection in humans as revealed by auditory brainstem and middlelatency evoked potentials. Eur J Neurosci 2010 Sep;32(5):859-65. doi: 10.1111/j.1460-9568.2010.07324.x.

15. Doi M, Morita K, Mantzaridis H, Sato S, Kenny GN. Prediction of responses to various stimuli during sedation: a comparison of three EEG variables. Intensive Care Med 2005 Jan;31(1):41-7.

16. Doi M, Gajraj RJ, Mantzaridis H, Kenny GN. Relationship between calculated blood concentration of propofol and electrophysiological variables during emergence from anaesthesia: comparison of bispectral index, spectral edge frequency, median frequency and auditory evoked potential index. Br J Anaesth 1997 Feb;78(2):180-4.

17. Stoppe C, Peters D, Fahlenkamp AV, Cremer J, Rex S, Schälte G, et al. aepEX monitor for the measurement of hypnotic depth in patients undergoing balanced xenon anaesthesia. Br J Anaesth 2012 Jan;108(1):80-8. doi: 10.1093/bja/aer393.

18. Hayakawa K, Tasaki O, Hamasaki T, Sakai T, Nakagawa Y, Ogura H, et al. Prognostic indicators and outcome prediction model for patients with return of spontaneous circulation from cardiopulmonary arrest: the Utstein Osaka Project. Resuscitation 2011 Jul;82(7):874-80. doi: 10.1016/j/resuscitation.2011.02.045.

19. Marko N. Hypothermia during percutaneous coronary intervention in comatose survivors of cardiac care. SIGNA VITAE 2010;5 suppl 1:13-6.

20. Wampler DA, Collett L, Manifold CA, Velasquez C, McMullan JT. Cardiac arrest is rare without prehospital return of spontaneous circulation. Prehosp Emerg Care 2012 Oct-Dec;16(4):451-5. doi: 10.3109/10903127.2012.695435. 